\title{
When Galaxies Collide: The Search for Low-frequency Gravitational Wave Backgrounds in the Universe
}

\author{
Sydney J. Chamberlin
}

The Leonard E. Parker Center for Gravitation, Cosmology \& Astrophysics Department of Physics, University of Wisconsin-Milwaukee Milwaukee, WI USA

\section{Introduction}

Gravitational waves (GWs) are a predicted feature of General Relativity (GR). These waves, which are tiny ripples in the fabric of space-time, appear as wavelike solutions to the vacuum Einstein equation, and are the only prediction from GR that have not yet been directly observed. In the past few decades, large scale efforts aimed at detecting these elusive waves have increasingly gained momentum: a direct observation of GWs will provide an entirely new mechanism for learning more about the structure and evolution of astrophysical objects in our universe, as well as the cosmology that underlies the universe itself.

Gravitational radiation may be generated by a number of interesting astrophysical and cosmological sources. Compact objects (specifically, asymmetric or bumpy rotating objects) such as neutron stars, supernovae, and mergers of compact binary objects are just a few of the potential sources of this radiation. In this work, we are interested in low-frequency GWs $\left(10^{-9} \mathrm{~Hz}-10^{-6} \mathrm{~Hz}\right)$ which are thought to be generated primarily by supermassive black hole binaries (SMBHBs) with masses $\left(M \gtrsim 10^{9} M_{\odot}\right)$ Jaffe and Backer [2003], Sesana et al. [2008, 2009].

In this presentation, we describe the type of GW signal that we are searching for, and explain how pulsars may be used to aid in the detection of this signal. We present a package of software algorithms and tools that we have extensively developed to use for our detection efforts, and point out some future tasks that need to be accomplished. For additional details, readers are encouraged to keep an eye out for our upcoming publication on this work (Chamberlin et al.).

\section{Detecting GW backgrounds with pulsar timing arrays}

Millisecond pulsars are among the most stable clocks in the universe. Observations of the radio signals being emitted by these pulsars are in fact so precise that they can be more accurate than an atomic clock. The high precision of pulsar timing observations is what allows pulsars to be used in GW searches: a GW, if present between the Earth and a pulsar,

The author extends thanks to the Wisconsin Space Grant Consortium for funding this work through a graduate fellowship. 


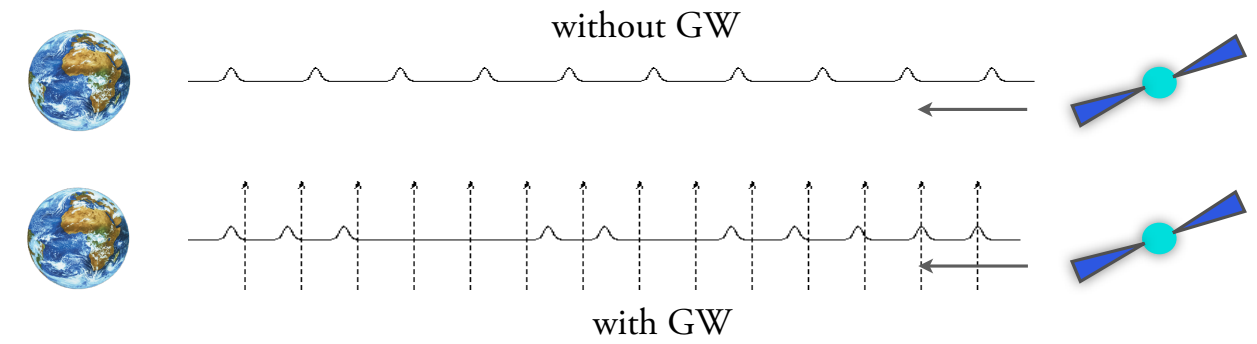

Figure 1: Two images showing the travel of a pulse-train from the pulsar to the Earth. In the top image, no GWs are present and the signals are timed as precisely as at any other time. In the lower image, dashed arrows represent areas in which a passing GW could cause a redshift in the pulse-train, and cause signals to arrive delayed or early at the Earth.

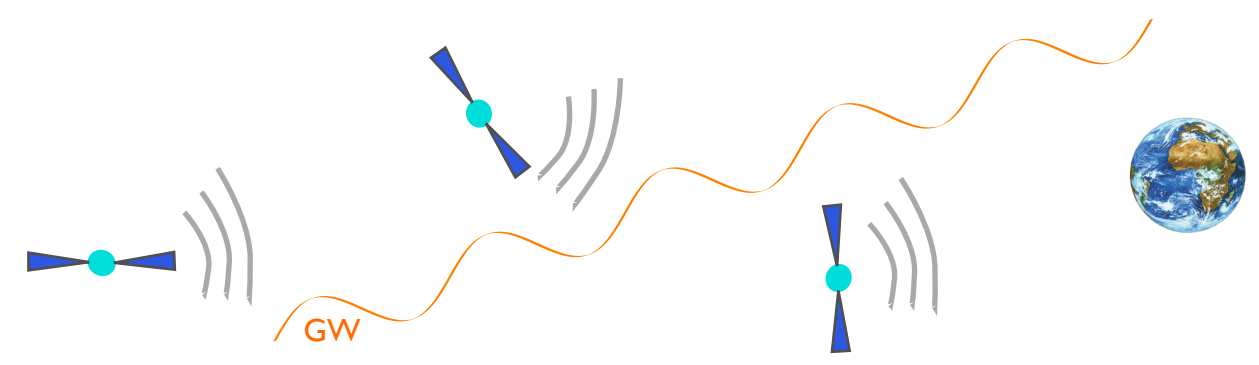

Figure 2: This figure demonstrates how a passing GW will effect the pulse-train from multiple pulsars. This effect will be correlated in a very unique way, allowing for a GW detection to take place.

will cause a redshift in the pulsar's radio pulse-train that is detectable at the Earth (see Fig. 1).

The quantity that is actually measured by radio astronomers is called the timing residual, which is defined as the difference between the actual and expected time-of-arrival (TOA) of the pulse at the Earth:

$$
r(t)=\mathrm{TOA}_{\text {actual }}-\mathrm{TOA}_{\text {expected }} .
$$

The observation of a single pulsar's timing residual $r(t)$ doesn't allow for any way to extract information about a passing GW signal, however, because it is impossible to tell whether a GW-induced redshift or some other effect is causing the delay of the signal. Instead of a single pulsar, an array of well-timed pulsars across the sky is used (hence the term pulsar timing array, or PTA). The idea, first proposed by Hellings and Downs [1983], is that the passing GW induces uniquely correlated changes in the pulsars' signals (see Fig. 2). Given an array of pulsars, therefore, a direct detection of GWs is possible. Several collaborations exist across the globe to take part in this effort: the North American Nanohertz Gravitational 
Wave Observatory (NANOGrav), the European Pulsar Timing Array (EPTA) and the Parkes Pulsar Timing Array (PPTA) together form an international collaboration, the International Pulsar Timing Array (IPTA) which is working towards a direct observation of GWs.

\section{The stochastic background}

In this work, we are not concerned with the gravitational radiation emitted by a single astrophysical source, but instead a bath of background gravitational radiation. Such a bath, or background, is caused not by a single source but by a very large number of independent and individually unresolvable sources. For our expected dominant source of GWs - SMBHBs - such background could form (for instance) from the collision of galaxies throughout the universe. Electromagnetic observations, such as those from the Hubble Deep Field, have made it apparent that a very large number of galaxies and galactic collisions have taken place over the universe's lifetime. The GWs emitted by each of these events together form a background of gravitational radiation, in much the same way as the Cosmic Microwave Background forms a background of electromagnetic radiation thoughout the universe.

This background of GWs is typically referred to as the stochastic GW background, and is the type of gravitational radiation that we seek to detect in this work.

\section{Detection algorithms}

This project has involved the development of statistical data analysis tools and techniques aimed at extracting the GWs (that are hopefully) present in pulsar timing data. The primary feature of the statistical analysis involves the unique Hellings and Downs correlation between pulsar signals and is called the optimal detection statistic. This statistic conveys the level of confidence that a GW signal is present in the data.

Starting with pulsar TOA data, the timing residuals $r(t)$ are determined. To make this transition, known effects (pulsar spin-down, interstellar medium effects, etc.) are taken into account using sophisticated software techniques. A series of Python and $\mathrm{C}$ codes then take the timing residual data from the PTAs, and calculate the optimal detection statistic. This

formalism also provides a robust method for generating frequentist upper limits in the data (see Fig. 3 for details on how this is accomplished). 


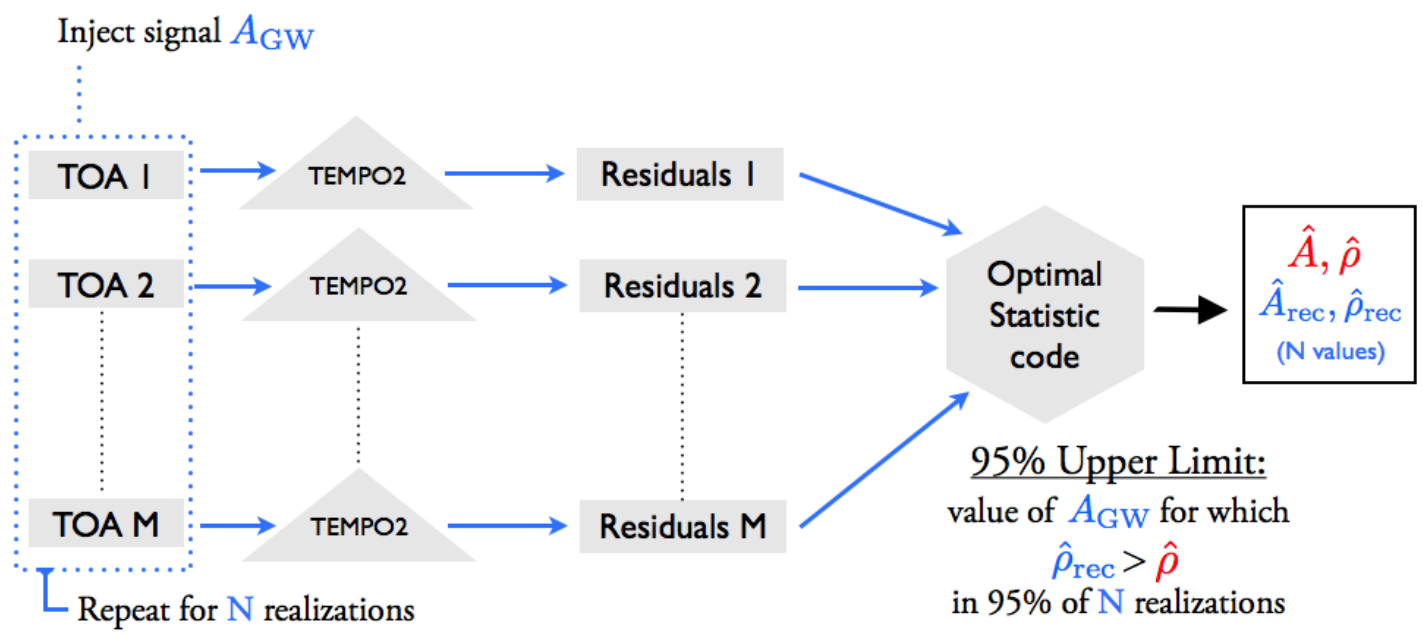

Figure 3: This schematic displays the elements of the stochastic GW detection pipeline. Assuming a PTA consisting of M pulsars, $\mathrm{M}$ timing residuals are obtained with the software Tempo2 Hobbs et al. [2006]. The $\mathrm{M}$ timing residuals then undergo a series of calculations resulting in a value for the detection statistic, $\hat{A}$, and signal-to-noise ratio $\hat{\rho}$. Examining this statistic gives a measure of confidence as to whether a signal is present in the data. Fake GW signals can then be injected into the data for some large number of realizations, N, and the same analysis performed for the data with injections to obtain $\hat{A_{\text {rec }}}$ and $\hat{\rho}_{\text {rec }}$. The $95 \%$ upper limits are obtained by finding the value of injected signal for which the recovered statistic $\hat{A}_{\text {rec }}$ exceeded the initial value of the statistic $\hat{A}$ in $95 \%$ of the realizations.

\section{Conclusion}

A direct detection of GWs will open a new observational window on the universe, and illuminate new science in a way that traditional electromagnetic observations cannot. Here, we have presented efforts aimed at detecting a stochastic GW background, which is generated by a large number of independent, individually unresolvable sources. We have discussed the manner in which pulsars may be used to detect GWs, and briefly introduced the software developments that we have undertaken to aid in GW detection efforts.

\section{References}

A. H. Jaffe and D. C. Backer. Gravitational Waves Probe the Coalescence Rate of Massive Black Hole Binaries. 583:616-631, February 2003. doi: 10.1086/345443.

A. Sesana, A. Vecchio, and C. N. Colacino. The stochastic gravitational-wave background from massive black hole binary systems: implications for observations with Pulsar Timing Arrays. 390:192-209, October 2008. doi: 10.1111/j.1365-2966.2008.13682.x. 
A. Sesana, A. Vecchio, and M. Volonteri. Gravitational waves from resolvable massive black hole binary systems and observations with Pulsar Timing Arrays. 394:2255-2265, April 2009. doi: 10.1111/j.1365-2966.2009.14499.x.

S. J. Chamberlin, J. D. E. Creighton, P. Demorest, J. Ellis, L. Price, and X. Siemens. A Practical Application of the Optimal Statistic for Stochastic Gravitational Wave Background Searches in Pulsar Timing Data. In Preparation; to be submitted to Physical Review D.

R. W. Hellings and G. S. Downs. Upper limits on the isotropic gravitational radiation background from pulsar timing analysis. 265:L39-L42, February 1983. doi: 10.1086/183954.

G. B. Hobbs, R. T. Edwards, and R. N. Manchester. TEMPO2, a new pulsar-timing package - I. An overview. 369:655-672, June 2006. doi: 10.1111/j.1365-2966.2006.10302.x. 